\title{
Temperature dependence of surface magnetization in local-moment systems
}

\author{
Alireza Saffarzadeh* \\ ${ }^{1}$ Department of Physics, Payame Noor University, Nejatollahi St., 159995-7613 Tehran, Iran \\ ${ }^{2}$ Computational Physical Sciences Research Laboratory, Department of Nano-Science, \\ Institute for studies in theoretical Physics and Mathematics (IPM), P.O. Box 19395-5531, Tehran, Iran
}

(Dated: October 28, 2018)

\begin{abstract}
We present a theory to study the temperature-dependent behavior of surface states in a ferromagnetic semi-infinite crystal. Our approach is based on the single-site approximation for the $s-f$ model. The effect of the semi-infinite nature of the crystal is taken into account by a localized perturbation method. Using the mean-field theory for the layer-dependent magnetization, the local density of states and the electron-spin polarization are investigated at different temperatures for ordinary and surface transition cases. The results show that the surface magnetic properties may differ strongly from those in the bulk and the coupling constant of atoms plays a decisive role in the degree of spin polarization. In particular, for the case in which the exchange coupling constant on the surface and between atoms in the first and second layer is higher than the corresponding in the bulk, an enhancement of surface Curie temperature and hence the spin polarization can be obtained.
\end{abstract}

\section{INTRODUCTION}

Knowledge of the spin-dependent electronic structure at surfaces and interfaces plays an increasingly important role when assessing possible use of novel magnetic materials for spintronic applications ${ }^{1}$. A surface breaks the translation symmetry of a system and changes local quantities such as local density of states (LDOS) which is significantly different from the bulk density of states (DOS). Experimental study on magnetic surfaces has shown that the order-disorder phase transitions are affected by the surfaces and may differ markedly from that in the bulk $k^{2,3,4,5,6}$. Indeed, the atoms at the surface layer have fewer neighboring atoms in comparison with the bulk, and it is well know that the magnetic order is strongly modified due to the lower coordination number. Moreover, the changes of the lattice constant near the magnetic surface also modify the exchange interaction constants. Therefore, the reduced translational symmetry may decrease or increase the magnetic stability.

Depending on the value of exchange coupling constant, two different behaviors at the surface can be obtained: (i) If the coupling constant is smaller than a critical valve, the bulk and surface ordering occur at the same temperature. This case is called ordinary transition. Experiments at the bulk Curie temperature have shown that in general case, the magnetization of thin films and surfaces is lower than the bulk one, and as the thickness of the films decreases, the magnetization also decreases. $\mathrm{Ni}$ and $\mathrm{Fe}$ correspond to this kind of systems 7.8 . (ii) If the exchange coupling constant is larger than a critical value, which is itself larger than the bulk coupling constant, the Curie temperature of thin films and surfaces is higher than the bulk Curie temperature. This case is called surface transition where the surface layer alone orders, while the bulk remains disordered. This remarkable phenomenon was observed for various compounds, such as for example $\mathrm{Gd}^{9,10}, \mathrm{~Tb}^{11}$ and $\mathrm{NiO}^{12,13}$. However, for Gd it should be mentioned that there are also newer studies claiming that no separate transition at the

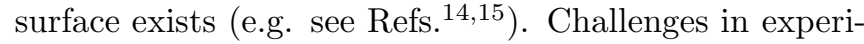
mental studies of surface magnetism stem from the difficulties of preparing and characterizing clean, structurally and magnetically ordered Gd surfaces.

From theoretical point of view, the spin-dependent band structure of films and surfaces have been investigated in local-moment systems in which the magnetic moment originates from a partially filled shell of the atoms and being strictly localized at each magnetic ion site. Kurz et al.16 studied the magnetism and the electronic structure of $\mathrm{Gd}(0001)$ surface on the basis of density functional theory. Schiller et al.17,18 investigated the temperature-dependent band structure of ferromagnetic semiconductor films and surfaces in the $s$ - $f$ mode ${ }^{19}$. Using a moment-conserving decoupling procedure for suitable defined Green's functions, the layer-dependent magnetizations and the LDOS were studied at different temperatures.

The aim of this work is to present the single-site coherent potential approximation (CPA) for the $s$ - $f$ to investigate the spin-polarized surface states in local-moment systems. To our knowledge, this is the first detailed study of the surface effects on electron-spin polarization using the CPA. In this work, based on the single-site CPA for the $s$ - $f$ model, we intend to develop a theory for electronic states of a magnetic semi-infinite crystal, which is applicable in wide range of temperature and the $s$ - $f$ exchange coupling strength.

The CPA is available when the scattering by the $s-f$ exchange interaction is equivalent to the scattering by a completely random short-ranged potential. In this approximation the multiple scattering on the single site is taken into consideration. The single-site CPA gives reasonable results for both weak and strong exchange interaction limits, and hence is fairly good for any values of bandwidth, scattering potential and temperature ${ }^{20}$. The single-site CPA, however, does not consider the exchange scattering caused by the $f$-spin correlation between different sites, which plays an important role around Curie temperature in local moment systems. In fact, the collec- 
tive mode, correlation, and/or clustering effect of localized spins are completely beyond the scope of the singlesite CPA.

The crystal is assumed to consist of completely filled and well ordered ferromagnetic layers and its surface is flat. Thus, the effects due to vacancies, islands, steps or intermixings on the magnetic properties are not considered here. However, the effect of magnetic anisotropy will be taken into account in the values of coupling constant between atoms on the surface of crystal.

The article is organized as follows: In section II, by applying the single-site approximation to the $s$ - $f$ model, we present a theory to study the surface effects on the spindependent electronic states in a magnetic semi-infinite crystal. In appendix A, we obtain the Green's function for a nonmagnetic semi-infinite crystal and in appendix $\mathrm{B}$, a procedure is given for calculation of the layer-dependent magnetization in the frame work of the mean-field approximation. The results of numerical calculations of the self-consistent equations for the LDOS and the electron-spin polarization are discussed in section III for two cases: ordinary and surface transitions. In section IV, we conclude the article with a summary.

\section{MODEL AND FORMALISM}

We consider a magnetic semi-infinite crystal which can be described by a single-orbital tight-binding Hamiltonian with nearest-neighbor hopping $t$ on a simple cubic (s.c.) crystalline lattice with lattice constant $a$. The structure is obtained by stacking layers parallel to the free surface and we choose the (001) axis of the s.c. structure to be normal to these layers. This direction is called $z$ direction hereafter and the system occupies the half-space $z>0$. We use the $s-f$ model which is commonly considered as realistic for local-moment structures $19,21,22$. In this model the following Hamiltonian is used to describe the magnetic semi-infinite crystal:

$$
\begin{gathered}
H_{t}=H_{s}+H_{f}+H_{s f} \\
H_{s}=t \sum_{n, n^{\prime}=1}^{+\infty} \sum_{\mathbf{r}, \mathbf{r}^{\prime}, \sigma}|\mathbf{r}, n ; \sigma\rangle\left\langle\mathbf{r}^{\prime}, n^{\prime} ; \sigma\right| \\
H_{f}=-\sum_{n, n^{\prime}=1}^{+\infty} \sum_{\mathbf{r}, \mathbf{r}^{\prime}} J_{\mathbf{r} n, \mathbf{r}^{\prime} n^{\prime}} \mathbf{S}_{\mathbf{r}, n} \cdot \mathbf{S}_{\mathbf{r}^{\prime}, n^{\prime}} \\
H_{s f}=-I \sum_{n=1}^{+\infty} \sum_{\mathbf{r}, \sigma, \sigma^{\prime}}|\mathbf{r}, n ; \sigma\rangle\left(\sigma_{\sigma \sigma^{\prime}} \cdot \mathbf{S}_{\mathbf{r}, n}\right)\left\langle\mathbf{r}, n ; \sigma^{\prime}\right|
\end{gathered}
$$

Here, $H_{s}$ describes the conduction electrons as $s$ electrons and gives the electron transfer energy in which $|\mathbf{r}, n ; \sigma\rangle$ is an orbital with spin $\sigma(=\uparrow, \downarrow)$ at site $\mathbf{r}$ in the $x$ - $y$ plane and layer $n(=1,2, \cdots,+\infty)$. Each lattice point of the semi-infinite crystal is occupied by a localized magnetic moment, represented by a spin operator $\mathbf{S}_{\mathbf{r}, n}$. The direct exchange coupling between these localized moments is expressed by the Heisenberg Hamiltonian $H_{f}$ where $J_{\mathbf{r} n, \mathbf{r}^{\prime} n^{\prime}}$ is the exchange coupling constant. The problem with the simple Heisenberg model in the form (3) is that the atoms of the topmost surface layer have fewer neighboring atoms than those of the bulk; thus, the magnetic order is strongly modified by the coordination number. This point is considered in calculation of the layer-dependent magnetization. $H_{s f}$ is the $s$ - $f$ exchange interaction between the $s$-electron and the localized $f$-spins where $\sigma_{\sigma \sigma^{\prime}}$ is the component of the Pauli matrix and $I$ is the $s-f$ exchange coupling constant. In this work, we regard a $f$-spin as a classical spin since the magnitude of the localized spin on a magnetic ion $(\mathrm{S}=7 / 2)$ is pretty large while the exchange interaction strength $I S$ is kept finite. The summations in the above equations extend over the sites of the semi-infinite crystal.

The single-electron Hamiltonian is defined as

$$
H=H_{e f f}+V,
$$

where the effective Hamiltonian $H_{\text {eff }}$ which describes the effective medium is expressed as

$$
H_{e f f}=H_{s}+\sum_{n=1}^{+\infty} \sum_{\mathbf{r}, \sigma}|\mathbf{r}, n ; \sigma\rangle \S_{n \sigma}\langle\mathbf{r}, n ; \sigma|,
$$

and the perturbation term $V$ is written as

$$
\begin{aligned}
V & =H_{s f}-\sum_{n=1}^{+\infty} \sum_{\mathbf{r}, \sigma}|\mathbf{r}, n ; \sigma\rangle \AA_{n \sigma}\langle\mathbf{r}, n ; \sigma| \\
& =\sum_{n=1}^{+\infty} \sum_{\mathbf{r}}\left\{\sum _ { \sigma , \sigma ^ { \prime } } | \mathbf { r } , n ; \sigma \rangle \left[-I\left(\sigma_{\sigma \sigma^{\prime}} \cdot \mathbf{S}_{\mathbf{r}, n}\right)\right.\right. \\
& =\sum_{n=1}^{+\infty} \sum_{\mathbf{r}} v_{\mathbf{r}, n} .
\end{aligned}
$$

Here $B_{n \sigma}$ is the layer- and spin-dependent coherent potential and $v_{\mathbf{r}, n}$ is an isolated potential in site $\mathbf{r}$ of the $n$-th effective layer.

As in Ref $\stackrel{20}{2}$, we apply the condition that the average scattering of the $s$-electron by the single $f$-spin embedded in the effective medium is zero. Thus we define the singlesite $t$-matrix of the $s-f$ exchange interaction as

$$
t_{\mathbf{r}, n}=v_{\mathbf{r}, n}\left[1-P v_{\mathbf{r}, n}\right]^{-1}
$$

where $P(\omega)=G^{0}\left(\omega-\beta_{n \sigma}\right)$ is the effective Green's function and $G^{0}(\omega)$ is the Green's function of the nonmagnetic semi-infinite crystal. Here, $t_{\mathbf{r}, n}$ is the complete scattering associated with the isolated potential $v_{\mathbf{r}, n}$. 
Within the single-site CPA, the condition $\left\langle t_{\mathbf{r}, n}\right\rangle_{t h}=0$ for any $\mathbf{r}$ in each layer, leads to the spin-dependent effective medium where an electron is subjected to a coherent potential, $\beta_{n \uparrow}$ or $\beta_{n \downarrow}$, according to the electron spin orientation. The spin-dependent coherent potentials are energy $(\omega)$-dependent complex potentials and in this study described by the following equations ${ }^{20}$ :

$$
\begin{aligned}
& \beta_{n \uparrow}=\frac{F_{n \downarrow} I^{2} S(S+1)-I S\left(1+F_{n \downarrow} \beta_{n \downarrow}\right)\left(A_{n \uparrow} / B_{n \uparrow}\right)}{1+F_{n \downarrow}\left(B_{n \downarrow}-I\right)-F_{n \downarrow} I S\left(A_{n \uparrow} / B_{n \uparrow}\right)}, \\
& \beta_{n \downarrow}=\frac{F_{n \uparrow} I^{2} S(S+1)+I S\left(1+F_{n \uparrow} \beta_{n \uparrow}\right)\left(A_{n \downarrow} / B_{n \downarrow}\right)}{1+F_{n \uparrow}\left(B_{n \uparrow}-I\right)+F_{n \uparrow} I S\left(A_{n \downarrow} / B_{n \downarrow}\right)},
\end{aligned}
$$

where

$$
A_{n \sigma}=\left\langle\frac{S_{n}^{z} / S}{\left(1-F_{n \sigma} V_{n \sigma}\right)\left(1-F_{n-\sigma} U_{n-\sigma}\right)-F_{n \sigma} F_{n-\sigma} W_{n \sigma}}\right\rangle_{t h},
$$

$$
\begin{gathered}
B_{n \sigma}=\left\langle\frac{1}{\left(1-F_{n \sigma} V_{n \sigma}\right)\left(1-F_{n-\sigma} U_{n-\sigma}\right)-F_{n \sigma} F_{n}}\right. \\
V_{n \sigma}=-z_{\sigma} I S_{n}^{z}-\beta_{n \sigma}, \\
U_{n \sigma}=-z_{\sigma} I\left(S_{n}^{z}-z_{\sigma}\right)-\beta_{n \sigma}, \\
W_{n \sigma}=I^{2}\left[S(S+1)-S_{n}^{z}\left(S_{n}^{z}+z_{\sigma}\right)\right] .
\end{gathered}
$$

In above equations $\langle\cdots\rangle_{t h}$ means the thermal average, $z_{\uparrow}=+1, z_{\downarrow}=-1$, and

$$
\begin{aligned}
F_{n \sigma} \equiv F_{n \sigma}(\omega) & =\frac{1}{N_{\|}} \sum_{\mathbf{k}_{\|}} G_{n n}^{0}\left(\mathbf{k}_{\|} ; \omega-\beta_{n \sigma}\right) \\
& =\int_{-\infty}^{\infty} d \epsilon D^{(2)}(\epsilon) G_{n n}^{0}\left(\epsilon ; \omega-\beta_{n \sigma}\right)
\end{aligned}
$$

where $N_{\|}$is the number of sites per layer, $\mathbf{k}_{\|}$is a wave vector parallel to the layers, $D^{(2)}(\epsilon)$ is the density of state for a square lattice with only nearest-neighbor hopping, and $G_{n m}^{0}\left(\mathbf{k}_{\|}, \omega\right)$ is a matrix element of the Green's function of a semi-infinite crystal which is given in Appendix A.

It is clear that the Eqs. (13)-(17) include only $S_{n}^{z}$ as an $f$-spin operator. Therefore, the thermal average for fluctuating $f$-spins can be easily calculated using the molecular-field theory. On the other hand, the existence of surface modifies the magnetic order in semi-infinite crystals. Thus, the thermal average of the $f$-spin operator depends on the layer index $n$. In appendix B we have derived and depicted the normalized magnetizations $\left\langle S_{n}^{z}\right\rangle / S$ which depend on layer index.

However, with increasing $n$ from the surface layer, the eigenvalues of semi-infinite Hamiltonian rapidly approach the bulk Hamiltonian. We find that the localized energies within the surface layer $(n=1)$, or at most, in the second layer $(n=2)$, i.e. the first interior layer, differ from that in the bulk. Here, we approximate the layer-dependent coherent potential, $\beta_{n \sigma}$, by the layer-independent bulk one, $\beta_{\sigma}^{b}$, for all layers $(n \geq 1)$. The difference in the coherent potentials is treated as a local perturbation in the semi-infinite Hamiltonian and applies only for the layers $n=1$ and $n=2$. Therefore, we approximate the effective Hamiltonian (6) by the following simple form:

$$
\begin{gathered}
H_{e f f}=H_{e f f}^{0}+U, \\
H_{e f f}^{0}=H_{s}+\sum_{n=1}^{+\infty} \sum_{\mathbf{r}, \sigma}|\mathbf{r}, n ; \sigma\rangle \beta_{\sigma}^{b}\langle\mathbf{r}, n ; \sigma|, \\
U=\sum_{n=1}^{2} \sum_{\mathbf{r}, \sigma}|\mathbf{r}, n ; \sigma\rangle u_{n \sigma}\langle\mathbf{r}, n ; \sigma|,
\end{gathered}
$$

'with

$$
u_{n \sigma}=\beta_{n \sigma}-\beta_{\sigma}^{b}
$$

Here, $u_{n \sigma}$ is a local perturbation for electrons with spin $\sigma$ in the $n$-th layer of the semi-infinite crystal.

The layer- and spin-dependent Green's function $\bar{G}_{n m \sigma}$ which corresponds to $H_{e f f}$ is then given by

$$
\begin{aligned}
\bar{G}_{n m \sigma}\left(\mathbf{k}_{\|} ; \omega\right) & =\bar{G}_{n m \sigma}^{0}\left(\mathbf{k}_{\|} ; \omega\right) \\
& +\sum_{l=1}^{2} \bar{G}_{n l \sigma}^{0}\left(\mathbf{k}_{\|} ; \omega\right) u_{l \sigma} \bar{G}_{l m \sigma}\left(\mathbf{k}_{\|} ; \omega\right),
\end{aligned}
$$

where

$$
\bar{G}_{1 m \sigma}=\frac{\bar{G}_{1 m \sigma}^{0}\left[1-\bar{G}_{22 \sigma}^{0} u_{2 \sigma}\right]+\bar{G}_{12 \sigma}^{0} u_{2 \sigma} \bar{G}_{2 m \sigma}^{0}}{\left[1-\bar{G}_{11 \sigma}^{0} u_{1 \sigma}\right]\left[1-\bar{G}_{22 \sigma}^{0} u_{2 \sigma}\right]-\bar{G}_{12 \sigma}^{0} u_{2 \sigma} \bar{G}_{21 \sigma}^{0} u_{1 \sigma}},
$$

$$
\bar{G}_{2 m \sigma}=\frac{\bar{G}_{2 m \sigma}^{0}\left[1-\bar{G}_{11 \sigma}^{0} u_{1 \sigma}\right]+\bar{G}_{21 \sigma}^{0} u_{1 \sigma} \bar{G}_{1 m \sigma}^{0}}{\left[1-\bar{G}_{11 \sigma}^{0} u_{1 \sigma}\right]\left[1-\bar{G}_{22 \sigma}^{0} u_{2 \sigma}\right]-\bar{G}_{12 \sigma}^{0} u_{2 \sigma} \bar{G}_{21 \sigma}^{0} u_{1 \sigma}}
$$

and $\bar{G}_{n m \sigma}^{0}\left(\mathbf{k}_{\|} ; \omega\right)=G_{n m}^{0}\left(\mathbf{k}_{\|} ; \omega-\beta_{\sigma}^{b}\right)$ is the corresponding Green's function of $H_{e f f}^{0}$ and can be obtained from appendix A. Therefore, using Eqs. (23)-(25) the diagonal elements of the Green's function for the semi-infinite effective crystal can be resulted from the relation

$$
\bar{F}_{n \sigma} \equiv \bar{F}_{n \sigma}(\omega)=\int_{-\infty}^{\infty} d \epsilon D^{(2)}(\epsilon) \bar{G}_{n n \sigma}(\epsilon ; \omega)
$$

However, before determining $\bar{F}_{n \sigma}$, we should obtain $\beta_{\sigma}^{b}$. In Eqs. (11)-(17), by changing $\beta_{n \sigma} \rightarrow \beta_{\sigma}^{b}, B_{n \sigma} \rightarrow B_{\sigma}$, 


$$
\begin{aligned}
A_{n \sigma} \rightarrow A_{\sigma}, V_{n \sigma} & \rightarrow V_{\sigma}, U_{n \sigma} \rightarrow U_{\sigma}, W_{n \sigma} \rightarrow W_{\sigma}, \text { and } \\
F_{n \sigma} \rightarrow F_{\sigma}^{b}(\omega) & =\frac{1}{N} \sum_{\mathbf{k}} \frac{1}{\omega-\beta_{\sigma}^{b}(\omega)-\epsilon(\mathbf{k})} \\
& =\int_{-\infty}^{\infty} d \epsilon D^{(2)}(\epsilon) \frac{-3 i}{W \sqrt{1-9\left(\frac{\omega-\beta_{\sigma}^{b}-\epsilon}{W}\right)^{2}}}
\end{aligned}
$$

we can obtain the bulk self-energy 20 . Here $F_{\sigma}^{b}$ is the diagonal element of the bulk effective Green's function, $N$ is the number of cells in the infinite crystal, and $\epsilon(\mathbf{k})=$ $2 t\left(\cos k_{x} a+\cos k_{y} a+\cos k_{z} a\right)$.

When $\beta_{\sigma}^{b}$ is determined, we can obtain $\beta_{n \sigma}$ using Eqs. (11)-(17). However, in these equations we use of $\bar{F}_{n \sigma}$ (Eq. (26)) instead of $F_{n \sigma}$ (Eq. (18)). This procedure is repeated until the calculation converges. After calculating the layer- and spin-dependent Green's function $\bar{F}_{n \sigma}$, the LDOS in layer $n$ can be determined by the relation

$$
D_{n \sigma}(\omega)=-\frac{1}{\pi} \operatorname{Im} \bar{F}_{n \sigma}(\omega+i \delta),
$$

which should satisfy the following equation in all of the present numerical calculations

$$
\int_{-\infty}^{\infty} D_{n \sigma}(\omega) d \omega=1
$$

In Eq. (28), $\delta$ is a positive infinitesimal.

In order to study the electron-spin polarization, we assume that $N_{\uparrow} / N_{\downarrow}$ is equal to $D_{n \uparrow}(\omega) / D_{n \downarrow}(\omega)$ where $N_{\uparrow}\left(N_{\downarrow}\right)$ is the number of electrons with spin-up (down). Thus the degree of electron-spin polarization at Fermi energy $\omega_{F}$ and in layer $n$ can be given by

$$
P_{n}\left(\omega_{F}, T\right)=\frac{D_{n \uparrow}\left(\omega_{F}\right)-D_{n \downarrow}\left(\omega_{F}\right)}{D_{n \uparrow}\left(\omega_{F}\right)+D_{n \downarrow}\left(\omega_{F}\right)} .
$$

\section{RESULTS AND DISCUSSION}

Using the approach described in the previous section, we have studied the spin-polarized surface states for $I S / W=0.35$ which corresponds to the intermediate $s$ $f$ exchange interaction. First we investigate the spindependent electronic states in different positions.

In Fig. 1, we have shown the DOS as a function of energy for a s.c. crystal at $T=0,0.5,0.8 T_{c}^{b}$, and $T \geq T_{c}^{b}$. It is clear that the largest spin-splitting between both spin-up and spin-down bands occurs at zero temperature. At this temperature, the spin-up DOS is similar to the DOS of a nonmagnetic crystal. Since at zero temperature (completely ferromagnetic case) all the local moments are aligned at $z$-direction, the magnetic system is periodic. As a result, the spin-up states are only shifted to the low energy side with no damping, and diminish at high energies where the coupling of the states are mainly antiparallel. On the other hand, the spin-down electrons

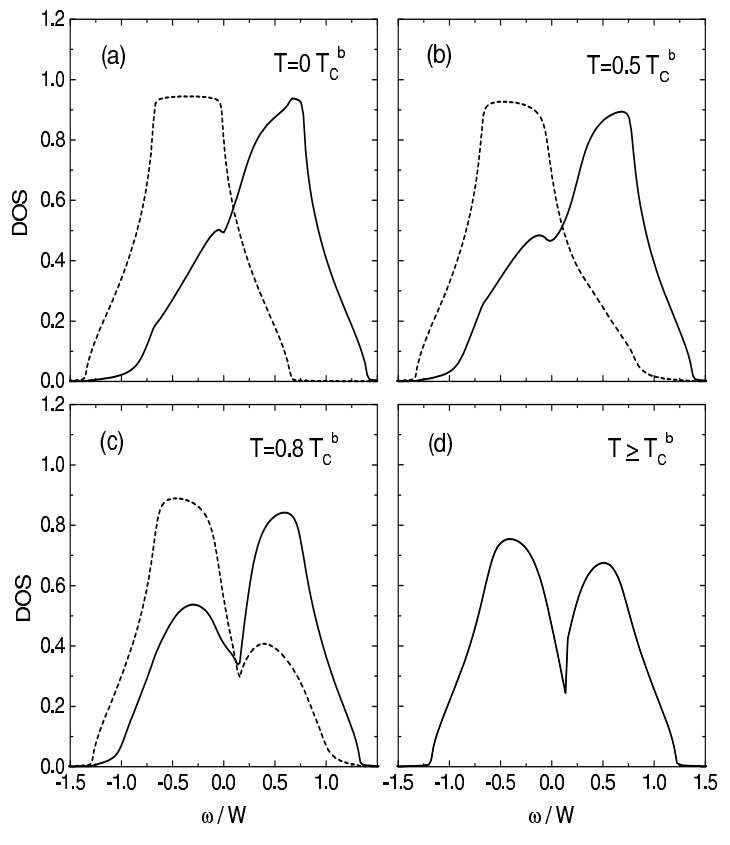

FIG. 1: The bulk DOS as a function of energy in various temperatures. The dashed (solid) curve is the DOS for spinup (spin-down) electrons.

can be flipped; thus, the spin-down band is shifted to the high energy region, while the bottom of the spin-down band extends down. With increasing the temperature, the local moments fluctuate and the spin-splitting between both spin bands decreases. At $T \geq T_{c}^{b}$, the spontaneous magnetization is zero and hence the DOS are spin-independent.

In Figs. 2 and 3, we have depicted the surface LDOS for spin-up and spin-down electrons at different temperatures. The curves in Fig. 2 correspond to the case in which the surface orders at a temperature $T_{C}^{s}$ (surface transition) larger than the bulk transition temperature $T_{C}^{b}$. In this regard, we have chosen $J_{\|}=1.5 \mathrm{~J}$ and $J_{\perp}=1.15 \mathrm{~J}$. The curves in Fig. 3, however correspond to the case in which both the bulk and the surface order at identical temperature (ordinary transition). In such case we have assumed that $J_{\|}=0.8 J$ and $J_{\perp}=0.95 \mathrm{~J}$. If we consider the layer-dependent magnetization in Fig. 8, we will see that, at low temperatures, the LDOS in the case of surface transition is similar to those obtained in the case of ordinary transition. At zero temperature, the spin-up LDOS is similar to the surface LDOS in a nonmagnetic semi-infinite crystal23. With increasing temperature from zero to $T \simeq 0.5 T_{C}^{b}$, the LDOS does not change considerably in both cases. The spin-splitting in the LDOS is a measure of the electron-spin polarization which depends on the layer index. Further increase of temperature, decreases the spin-splitting of the LDOS. Fig. 2 shows that, for the surface transition the spinsplitting is not zero at $T=T_{C}^{b}$ and even at $T=1.1 T_{C}^{b}$, however for the ordinary transition the spin-splitting will be zero at $T=T_{C}^{b}$ as shown in Fig. 3 . 


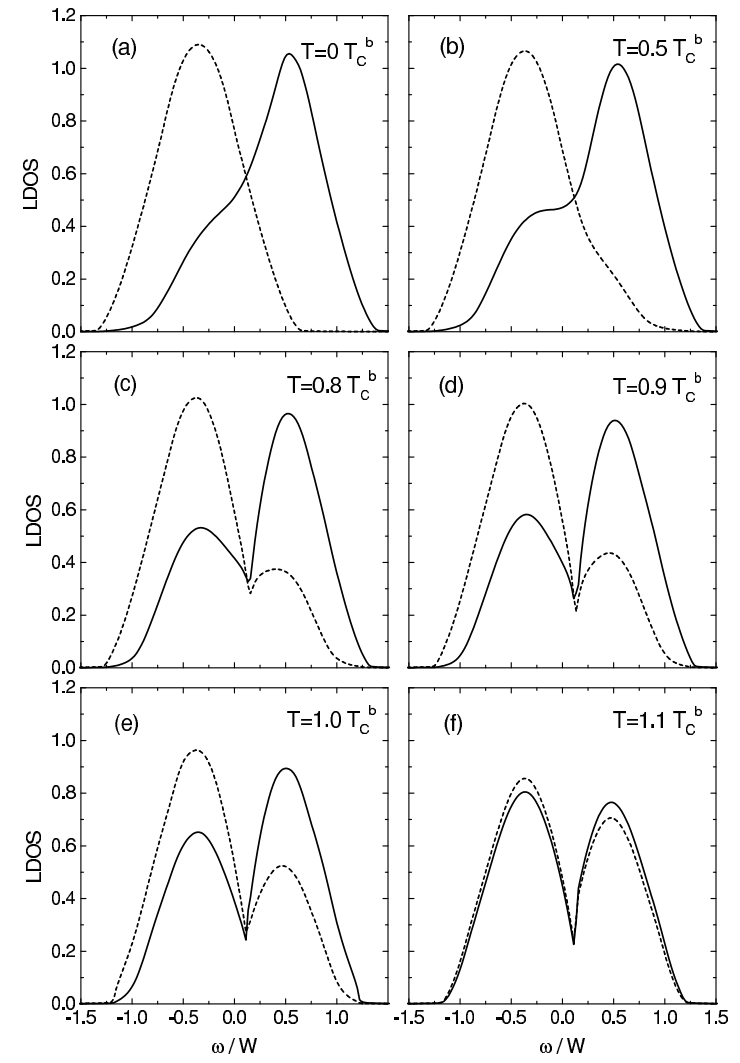

FIG. 2: The LDOS for the surface layer $(n=1)$ as a function of energy in various temperatures. The dashed (solid) curve is the LDOS for spin-up (spin-down) electrons. Here, $J_{\|}=1.50 \mathrm{~J}$ and $J_{\perp}=1.15 \mathrm{~J}$.

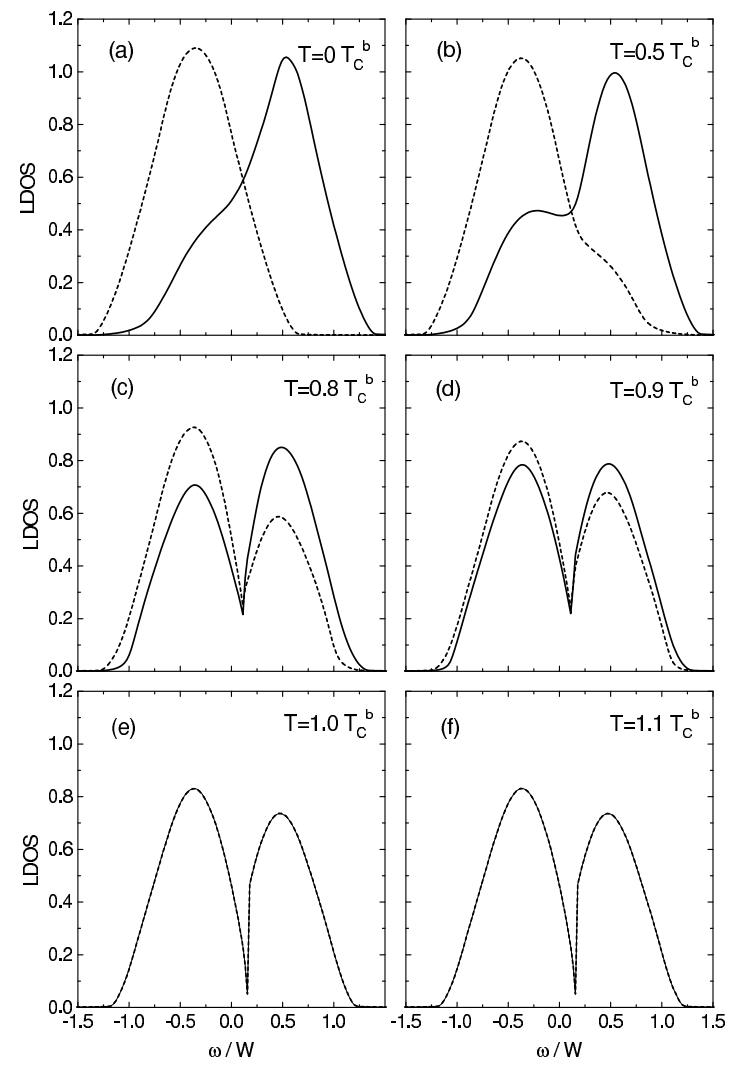

FIG. 3: The LDOS for the surface layer $(n=1)$ as a function of energy in various temperatures. The dashed (solid) curve is the LDOS for spin-up (spin-down) electrons. Here, $J_{\|}=0.80$ $J$ and $J_{\perp}=0.95 \mathrm{~J}$.

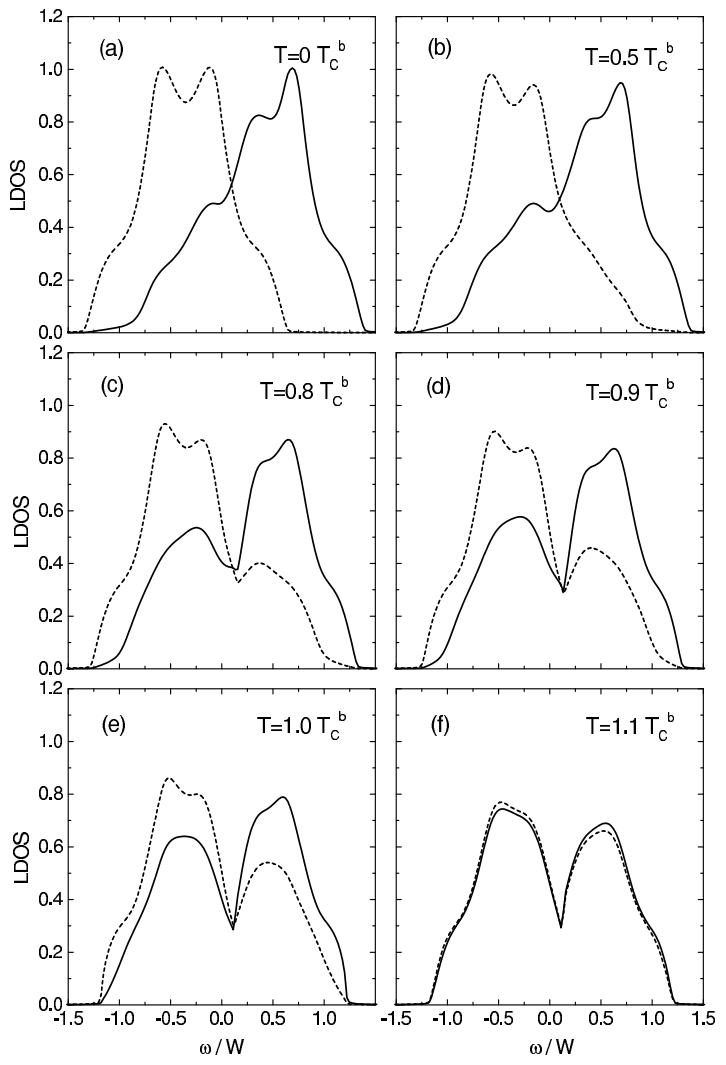

FIG. 4: The LDOS for the first interior layer $(n=2)$ as a function of energy in various temperatures. The dashed (solid) curve is the LDOS for spin-up (spin-down) electrons. Here, $J_{\|}=1.50 \mathrm{~J}$ and $J_{\perp}=1.15 \mathrm{~J}$.

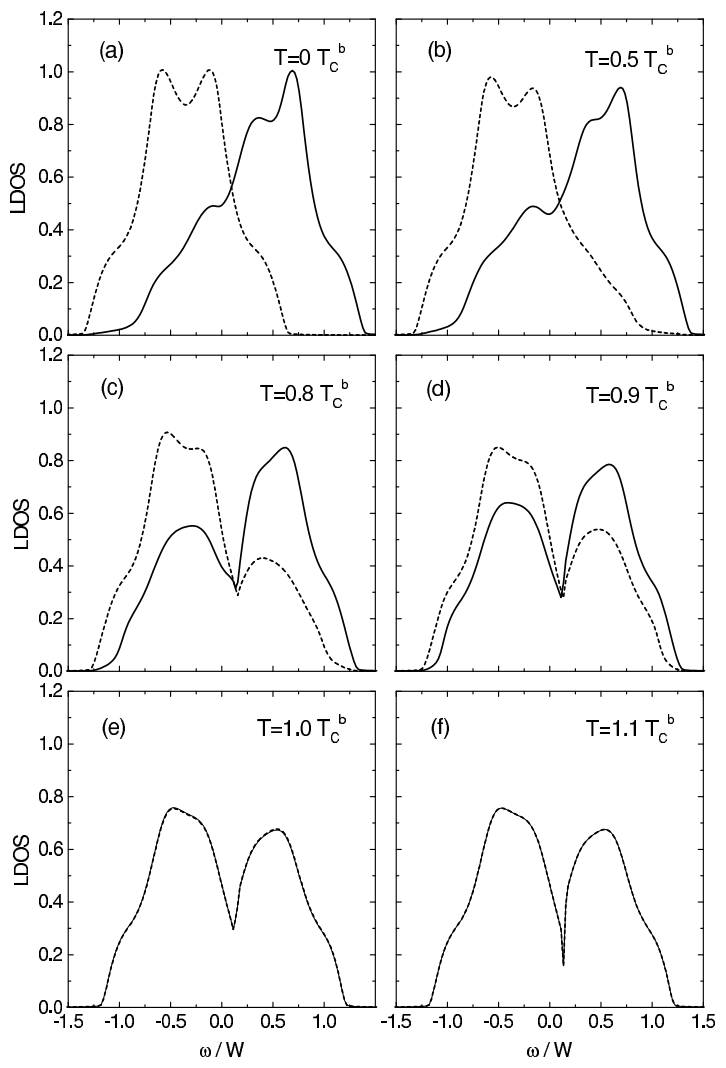

FIG. 5: The LDOS for the first interior layer $(n=2)$ as a function of energy in various temperatures. The dashed (solid) curve is the LDOS for spin-up (spin-down) electrons. Here, $J_{\|}=0.80 \mathrm{~J}$ and $J_{\perp}=0.95 \mathrm{~J}$. 


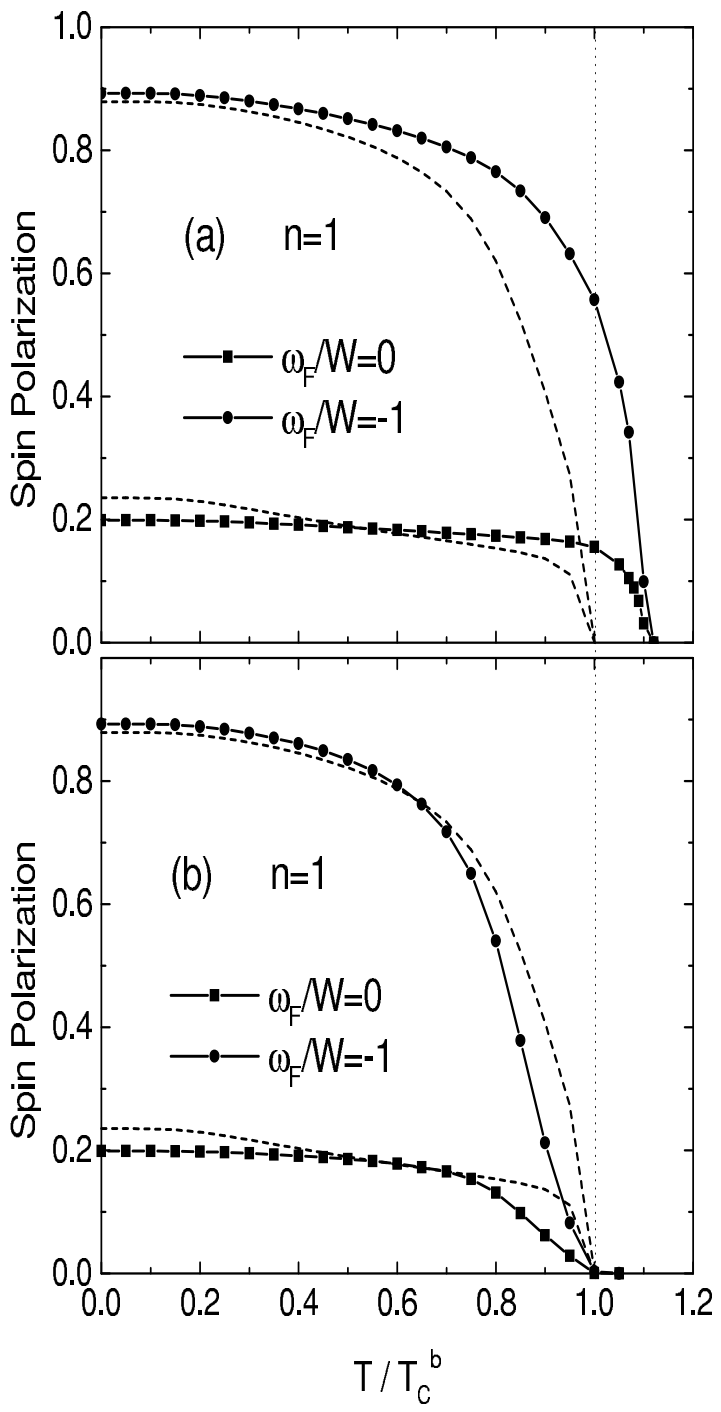

FIG. 6: The degree of spin polarization for the surface layer $(n=1)$ as a function of normalized temperature $T / T_{C}^{b}$ : (a) $J_{\|}=1.50 \mathrm{~J}$ and $J_{\perp}=1.15 \mathrm{~J}$, (b) $J_{\|}=0.80 \mathrm{~J}$ and $J_{\perp}=0.95 \mathrm{~J}$. The dashed curve is the bulk electron-spin polarization.

In Figs. 4 and 5 we have depicted the temperature and spin dependence of LDOS in the second layer at the surface and ordinary transition cases, respectively. The electronic states slightly resemble the DOS function for the infinite crystal. In this layer the spin-down band, as the previous electronic states, has a tail which reaches the edge of the spin-up band even at low temperatures. As the temperature increases, the spin-up (-down) LDOS shifts to high (low) energy side; so that, at paramagnetic region the LDOS for both spin-up and spin-down bands completely coincide to each other. It is clear that in all cases, there is a cusp in the LDOS which is due to the $s-f$ exchange coupling strength.

If we compare the Figs. 2(f) and $4(\mathrm{f})$ (at $T=1.1 T_{C}^{b}$ ), we will find that the value of spin-splitting at the surface layer is slightly more than the first interior layer (second

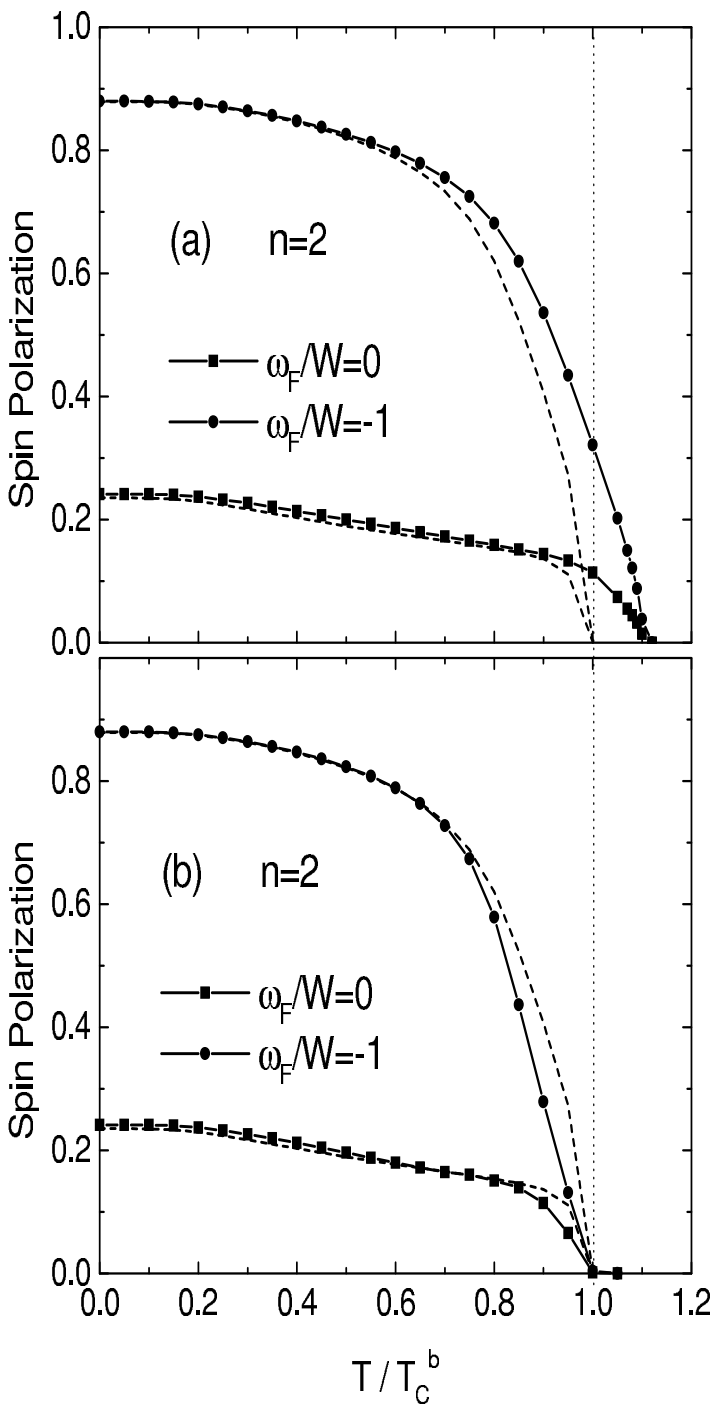

FIG. 7: The degree of spin polarization for the first interior layer $(n=2)$ as a function of normalized temperature $T / T_{C}^{b}$ : (a) $J_{\|}=1.50 \mathrm{~J}$ and $J_{\perp}=1.15 \mathrm{~J}$, (b) $J_{\|}=0.80 \mathrm{~J}$ and $J_{\perp}=0.95$ $J$. The dashed curve is the bulk electron-spin polarization.

layer). Therefore, as one proceeds into the crystal, the value of spin polarization begins to decrease at temperatures higher than $T_{C}^{b}$. When we are far enough from the surface, the surface effect on the spin polarization completely disappears and at $T \geq T_{C}^{b}$ the electron-spin polarization becomes zero.

In order to gain further insight into the surface effects on the LDOS, we have shown in Figs. 6 and 7, the temperature dependence of the electron-spin polarization in both surface and ordinary transition cases for the first and second layers, respectively. In most of the magnetic phenomena at surfaces, interfaces and in thin films, such as spin-polarized electron emission and spinpolarized transport through a magnetic film, the electrons at the Fermi surface play an essential role. Therefore, the electron-spin polarization depends on the posi- 
tion of Fermi energy. Here, in both Fig. 6 and 7, we have calculated the spin polarization at $\omega_{F} / W=-1$ (near the band edge) and at $\omega_{F} / W=0$ (near the band center). For comparison, we have shown the electronspin polarization for the infinite crystal. It is clear that, in surface transition case when $\omega_{F} / W=-1$, the spin polarization on the surface $(n=1)$ and in the first interior layer $(n=2)$ is higher than the bulk one. The spin polarization on the surface layer differs with the bulk one at all temperatures. This difference near $T_{C}^{b}$ reaches its maximum value. However, due to the reduction of the spin polarization in the first interior layer in comparison with the surface layer, the difference in the spin polarization of this layer and the bulk appears above $0.6 T_{C}^{b}$.

The difference of the spin polarizations between both layers and the bulk is originated from the strong exchange coupling with magnetic ions. At $\omega_{F} / W=0$, the spin polarizations have low values at all temperatures, because the difference between two spin bands is small. If we consider the ordinary transition case, we will see that, near $T_{C}^{b}$ that the surface magnetization has the most difference with the bulk one, the bulk spin polarization is more than the value of spin polarization at the layers $n=1$ and $n=2$.

We applied the present model for s.c. local moment systems such as Eu chalcogenides. This model is also applicable to the case of hcp Gd. However, for an atom at the $\operatorname{Gd}(0001)$ surface, we have six nearest-neighbor atoms at the surface, and three nearest-neighbor atoms in the second layer. Applying the molecular-field theory to the case of a hcp(0001) surface, we find that, for an exchange coupling ratio that satisfies $J^{s} \geq \frac{4}{3} J$, there is a surface transition 24 . Here, $J^{s}$ is the exchange coupling constant at the surface.

It should be noted that, real surfaces are usually not perfectly smooth and display some degree of roughness due to the presence of surface defects, such as for example steps, islands, or vacancies. Impurities are also often encountered at crystalline surfaces and may be viewed as the source of some disorder at the surface. All these defects have some impact on magnetic surface quantities. Based on the single-site CPA, Hong ${ }^{25}$ studied the LDOS near a rough surface. The numerical results show that, the LDOS depend sensitively on surface roughness. On the other hand, Zhao et al. ${ }^{26}$ using Ising-model Monte Carlo simulations have shown that surface roughness strongly affects surface magnetization, not only changing the surface magnetic ordering temperature but also modifying the magnitude of magnetization in a complex fashion. Therefore, both the LDOS and the surface magnetization are sensitive to the surface defects and consequently the resulting spin polarization is strongly influenced by the vacancies, islands, steps and the other surface defects.

The above results show that the exchange coupling between the surface magnetic ions plays an important role in the degree of spin polarization for the emitted electrons from the free surface or the transmitted electrons through interfaces. Hence, in designing the spin electronic or "spintronic" devices the information about the surface effects on the degree of spin-polarization plays an essential role in generation of highly polarized electrons.

\section{CONCLUSIONS}

In summary, we have presented a new formalism to study the spin-polarized surface states in the local moment systems. Based on the single-site CPA for the $s$ - $f$ model and the mean-field theory for the layer-dependent magnetization we have investigated how the surface influences the spin-dependent electronic states. The effect of the semi-infinity of the crystal was taken into account by a localized perturbation method. Assuming a s.c. band structure for the $s$ electrons we obtained the numerical results for the LDOS and the electron-spin polarization for various temperatures and in two different behaviors at the surface; ordinary and surface transitions. These transitions depend on the values of the exchange coupling constants between atoms on the surface and between atoms in the layers $n=1$ and $n=2$. The results showed that in the surface transition case, in which the coupling constants are higher than the corresponding in the bulk, the surface Curie temperature $T_{C}^{s}$ lies well above the bulk Curie temperature. Therefore, at $T_{C}^{b}<T<T_{C}^{s}$ the electron-spin polarization on the surface has a finite value, while the bulk spin polarization is zero. The existence of the surface magnetization and hence, the spin-polarized surface states above the bulk Curie temperature can be useful for development of new electron-polarized devices.

\section{Acknowledgments}

This work was supported by the Payame Noor University.

\section{APPENDIX A: GREEN'S FUNCTION OF A SEMI-INFINITE CRYSTAL}

The existence of surface affects the electronic eigenfunction; therefore, due to the surface effect, the Green's function of a semi-infinite crystal is essentially layerdependent. In this regard, for the eigenfunction of a semi-infinite crystal we use of a simple form as:

$$
\left|\mathbf{k}_{\|}, k_{\perp}\right\rangle=\frac{1}{\sqrt{N_{\perp}}} \sum_{n} \varphi_{n}\left(k_{\perp}\right)\left|\mathbf{k}_{\|}, n\right\rangle
$$

where the layer-dependent coefficient $\varphi_{n}\left(k_{\perp}\right)$ is given by the standing wave

$$
\varphi_{n}\left(k_{\perp}\right)=\sqrt{2} \sin \left(k_{\perp} n a\right),
$$


and satisfies the following orthogonality conditions

$$
\begin{aligned}
& \sum_{n} \varphi_{n}^{*}\left(k_{\perp}\right) \varphi_{n}\left(k_{\perp}^{\prime}\right)=\delta_{k_{\perp}, k_{\perp}^{\prime}} \\
& \sum_{k_{\perp}} \varphi_{n}^{*}\left(k_{\perp}\right) \varphi_{n^{\prime}}\left(k_{\perp}\right)=\delta_{n, n^{\prime}} .
\end{aligned}
$$

Using Eqs. (A1) and (A2), we obtain the layerdependent Green's function for a semi-infinite crystal with a (001) surface as follows;

$$
G_{n m}^{0}\left(\mathbf{k}_{\|} ; \omega\right)=\frac{1}{N_{\perp}} \sum_{k_{\perp}} \frac{\varphi_{n}^{*}\left(k_{\perp}\right) \varphi_{m}\left(k_{\perp}\right)}{\omega-\epsilon_{\mathbf{k}_{\|}}-2 t \cos \left(k_{\perp} a\right)} .
$$

Here $N_{\perp}$ is the number of layers parallel to the surface and $\epsilon_{\mathbf{k}_{\|}}=2 t\left(\cos k_{x} a+\cos k_{y} a\right)$.

By transforming the sum over $k_{\perp}$ in Eq. A5 to an integral over $k_{\perp} a=\theta$, the Green's function in a mixed Bloch-Wannier representation for a semi-infinite crystal is then given as

$$
G_{n m}^{0}\left(\mathbf{k}_{\|} ; \omega\right)=\frac{-3 i}{W \sqrt{1-\eta^{2}}}\left[f(\eta)^{|n-m|}-f(\eta)^{|n+m|}\right],
$$

where $W=6 t$ is the half-bandwidth and

$$
\begin{gathered}
\eta=3\left(\omega-\epsilon_{\mathbf{k}_{\|}}\right) / W \\
f(\eta)=\eta-i \sqrt{1-\eta^{2}} .
\end{gathered}
$$

\section{APPENDIX B: LAYER-DEPENDENT MAGNETIZATION}

Here, we like to present a generalized molecular-field theory to calculate the layer-dependent magnetization for a magnetic semi-infinite crystal. Due to a wide range of physical effects near surfaces, surface properties may be significantly different from bulk properties $11,27,28,29$. These are partly taken into account by a deviation of exchange coupling constant $J_{\mathbf{r} n, \mathbf{r}^{\prime} n^{\prime}}$ in nearest neighbor approximation and in the neighborhood of surface. For simplicity, we assume in all calculations that only the coupling constants of the local magnetic moments at the surface, $J_{\mathbf{r} 1, \mathbf{r}^{\prime} 1}=J_{\|}$, and also between atoms in the surface and second layer, $J_{\mathbf{r} 1, \mathbf{r} 2}=J_{\perp}$, differ from the bulk exchange coupling constant $J_{\mathbf{r} n, \mathbf{r}^{\prime} n^{\prime}}=J$.

The molecular-field equations given by $\operatorname{Cottam}^{29}$ for spontaneous magnetizations of the semi-infinite crystals with s.c. structure and (001) orientation of the surface, are of the form

$$
\frac{\left\langle S_{1}^{z}\right\rangle}{S}=B_{s}\left[\frac{S}{2 \tau(S+1)}\left(4 \frac{J_{\|}}{J} \frac{\left\langle S_{1}^{z}\right\rangle}{S}+\frac{J_{\perp}}{J} \frac{\left\langle S_{2}^{z}\right\rangle}{S}\right)\right],
$$

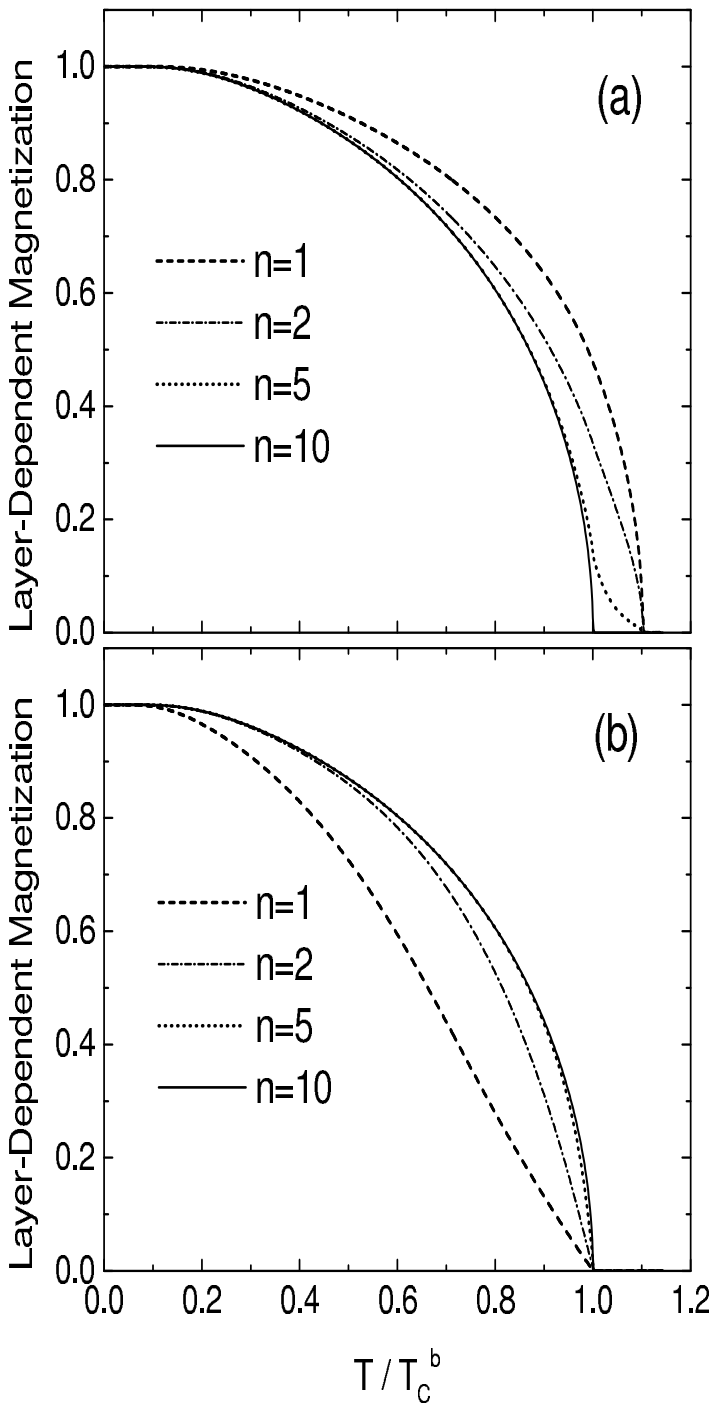

FIG. 8: Layer-dependent normalized magnetization, $\left\langle S_{n}^{z}\right\rangle / S$, as a function of normalized temperature, $T / T_{C}^{b}$. (a) $J_{\|}=1.50$ $J$ and $J_{\perp}=1.15 \mathrm{~J}$, (b) $J_{\|}=0.80 \mathrm{~J}$ and $J_{\perp}=0.95 \mathrm{~J}$.

$$
\begin{array}{r}
\frac{\left\langle S_{2}^{z}\right\rangle}{S}=B_{s}\left[\frac{S}{2 \tau(S+1)}\left(\frac{J_{\perp}}{J} \frac{\left\langle S_{1}^{z}\right\rangle}{S}+4 \frac{\left\langle S_{2}^{z}\right\rangle}{S}+\frac{\left\langle S_{3}^{z}\right\rangle}{S}\right)\right], \\
\frac{\left\langle S_{n}^{z}\right\rangle}{S}=B_{s}\left[\frac{S}{2 \tau(S+1)}\left(\frac{\left\langle S_{n-1}^{z}\right\rangle}{S}+4 \frac{\left\langle S_{n}^{z}\right\rangle}{S}+\frac{\left\langle S_{n+1}^{z}\right\rangle}{S}\right)\right] \\
n \geq 3, \text { (B3) }
\end{array}
$$

where $B_{s}(x)$ is the Brillouin function and $\tau=T / T_{C}^{b}$ in which $T_{C}^{b}$ is the bulk Curie temperature of the sample. Depending on the values of $J_{\|}$and $J_{\perp}$, two different behaviors at the surface can be obtained: (i) If the coupling constants $J_{\|}$and $J_{\perp}$ are larger than $J$, the magnetization at the surface and even in the layers near to the surface may have nonzero values at $T>T_{C}^{b}$. Therefore, these layers are in the ferromagnetic phase for $T_{C}^{b}<T<T_{C}^{s}$ ( $T_{C}^{s}$ is the surface Curie temperature), while the bulk is in paramagnetic state. (ii) If $J_{\|}$and $J_{\perp}$ are smaller 
than $J$, the surface and the layers near the surface may have weaker magnetization than the bulk at temperatures near $T_{C}^{b}$. The normalized magnetization in each case can be determined using the self-consistent solution of Eqs. (B1)-(B3). The layer-dependent magnetization for both cases has been shown in Fig. 8. It is important to note that, the temperature dependence of the magnetizations depends strongly on the boundary conditions imposed in the calculation. Here, the bulk boundary conditions were imposed in 10th layer ${ }^{30}$.
* E-mail: a-saffar@tehran.pnu.ac.ir

1 V.P. LaBella, D.W. Bullock, Z. Ding, C. Emery, A. Venkatesan, W.F. Oliver, G.J. Salamo, P.M. Thibado, and M. Mortazavi, Science 292, 1518 (2001).

2 N.C. Koon, B.T. Jonker, F.A. Volkening, J.J. Krebs, and G.A. Prinz, Phys. Rev. Lett. 59, 2463 (1987).

3 M. Stamspanoni, A. Vaterlaus, M. Aeschlimann, and F. Meier, Phys. Rev. Lett. 59, 2483 (1987).

4 C. Liu, E.R. Mong, and S.D. Bader, Phys. Rev. Lett. 60, 2422 (1988).

${ }^{5}$ W. Durr, M. Taborelli, O. Paul, R. Germar, W. Gudot, D. Pescia, and M. Landolt, Phys. Rev. Lett. 62, 206 (1989).

${ }^{6}$ C. Liu and S.D. Bader, J. Appl. Phys. 67, 5758 (1990).

7 R.J. Celotta, D.T. Pierce, G.C. Wang, S.D. Bader, and G.P. Felcher, Phys. Rev. Lett. 43, 728 (1979).

8 S.F. Alvarado, M. Campagna, and H. Hopster, Phys. Rev. Lett. 48, 51 (1982).

${ }^{9}$ D. Weller, S.F. Alvarado, W. Gudat, K. Schroder and M. Capagna, Phys. Rev. Lett. 54, 1555 (1985).

10 C. Rau, M. Robert, Phys. Rev. Lett. 58, 2714 (1987).

11 C. Rau, C. Jin, and M. Robert, J. Appl. Phys. 63, 3667 (1988)

12 M. Marynowski, W. Franzen, M. El-Batanouny, V. Staemmler, Phys. Rev. B 60, 6053 (1999).

13 M. El-Batanouny, J. Phys.: Condens. Matter 14, 6281 (2002).

14 M. Donath, B. Gubanka, F. Passek, Phys. Rev. Lett. 77, 5138 (1996).
15 C.S. Arnold, D.P. Pappas, Phys. Rev. Lett. 85, 5202 (2000).

16 Ph. Kurz, G. Bihlmayer, and S. Blügel, J. Phys.: Condens. Matter 14, 6353 (2002).

17 R. Schiller, W. Müller, and W. Nolting, J. Phys.: Condens. Matter 11, 9589 (1999).

18 R. Schiller and W. Nolting, Phys. Rev. B 60, 462 (1999).

19 E.L. Nagaev, Phys. Status Solidi B 65, 11 (1974).

20 M. Takahashi and K. Mitsui, Phys. Rev. B 54, 11298 (1996).

21 W. Nolting, Phys. Status Solidi B 96, 11 (1979).

22 S.G. Ovchinikov, Phase Transitions 36, 15 (1991).

23 D. Kalkstein and P. Soven, Surf. Sci. 26, 85 (1971).

24 A.B. Shick, W.E. Pickett, C.S. Fadley, Phys. Rev. B 61 R9213 (2000).

25 K.M. Hong, Solid State Commun. 66241 (1988).

26 D. Zhao, F. Liu, D.L. Huber, M.G. Lagally, Phys. Rev. B 6211316 (2000).

27 C. Rau and S. Eichner, Phys. Rev. B 34, 6347 (1986).

${ }^{28}$ H. Tang, D. Weller, T.G. Walker, J.C. Scott, C. Chappert, H. Hopster, A.W. Pang, D.S. Dessau, and D.P. Pappas, Phys. Rev. Lett. 71, 444 (1993).

29 M.G. Cottam and D.R. Tilly, Introduction to surface and superlattice excitations, Cambrdige, New Rochelle Melburne, Sydney, Cambridge University Press 1989.

30 J.L. Morán-López and J.M. Sanchez, Phys. Rev. B 39, 9746 (1989). 